\title{
Just a Little Bit
}

\section{Do you speak binary? Think about binary numbers and the sizes of the blocks in this puzzle. Your goal is to create a $Q R$ code!}

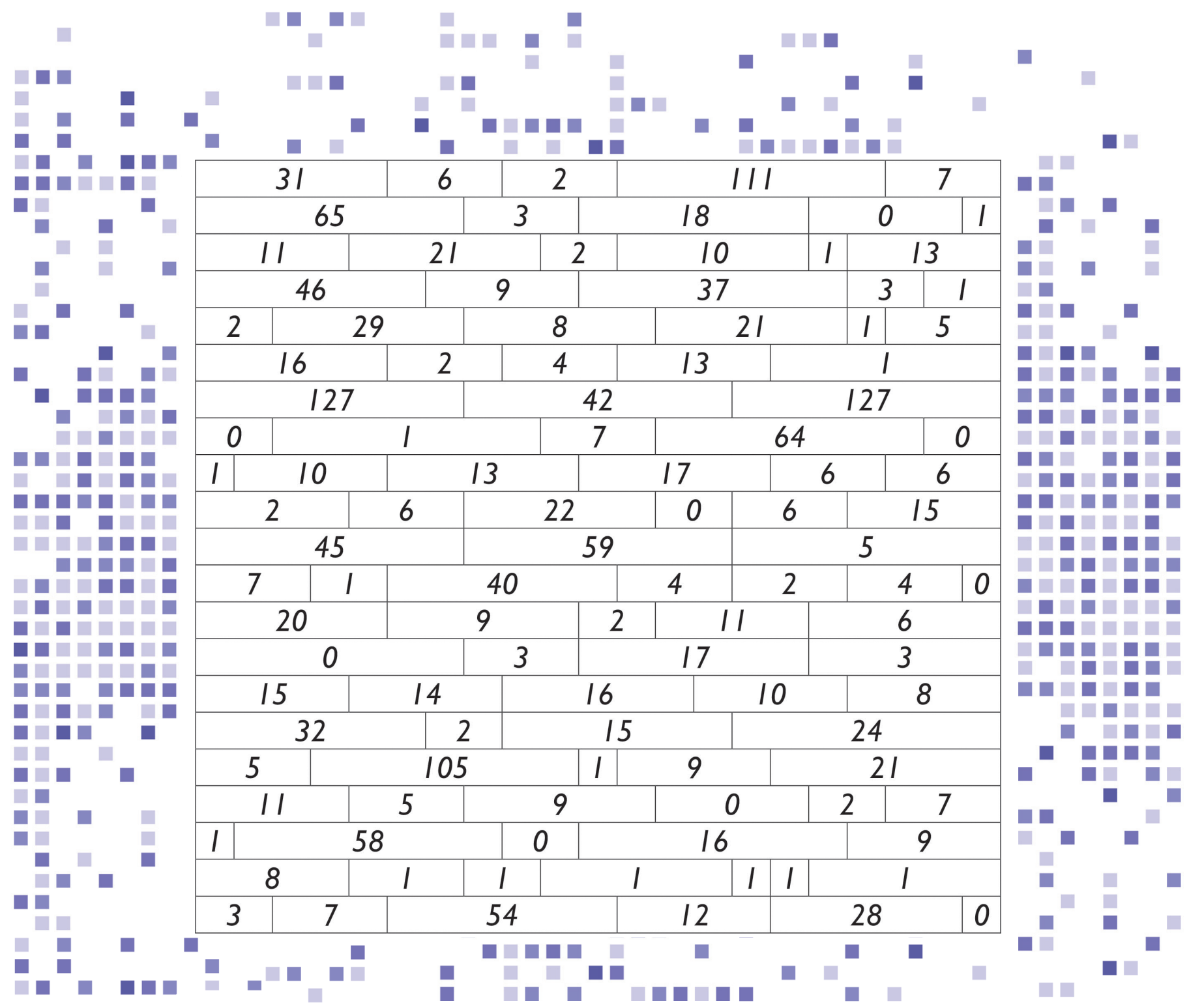

*CS50 is Harvard University's introduction to the intellectual enterprises of computer science and the art of programming. Each year, the course begins with CS50 Puzzle Day, an opportunity for students to collaborate with classmates on a packet of puzzles like this one. Among the event's goals is to send a message at term's start that CS isn't about programming but about problem solving more generally. Email outreach@cs50.harvard.edu if you'd like to organize a similar event!
Solution to the CS50* Puzzle Logic Gate from the March issue of acm INROADS. Solution: A R C H I T E C T U R E See: https://medium.com/@cs50/ this-was-cs50x-puzzle-day-201822d95a48615 